\title{
Applying Paints with Nitrogen
}

Companies in some countries already have experience of atomising paints using nitrogen. Tests carried out in one machinery manufacturer's technical centre have produced very positive results and these

have now been reinforced by the successful use of the process in volume production.

$\mathrm{O}$ ltrogge has been carrying out detailed studies into the atomisation of paints and other liquids with nitrogen for around two years. The nitrogen is produced from the existing compressed air network using a membrane system. Because of the concentrations and volume at which it is used, it presents no hazards to health. A nitrogen generator can be installed on a plugand-play basis with very little assembly work needed.

\section{Major benefits in the lab}

A number of complex tests of automatic atomisation were carried out in Oltrogge's technical centre to demonstrate the effects of nitrogen in different concentrations and levels of purity, combined with heat and ionisation, on the results of the painting process. These showed that the amount of materials used can be reduced significantly, regardless of whether solvents, water-based paints, high-solid products or release agents are being applied. The savings ranged between $5 \%$ and $45 \%$.

The tests also showed that the process offered further benefits, which, when investigated in greater detail, often prove to be more important and

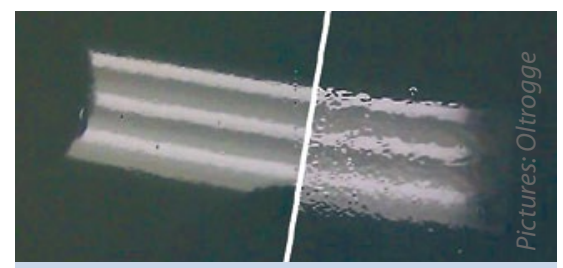

Nitrogen (left) versus compressed air (right): The picture on the left shows the first spraying attempt with the atomiser pressure reduced by half and without finetuning the application parameters. The coating applied was around $40 \%$ thicker.

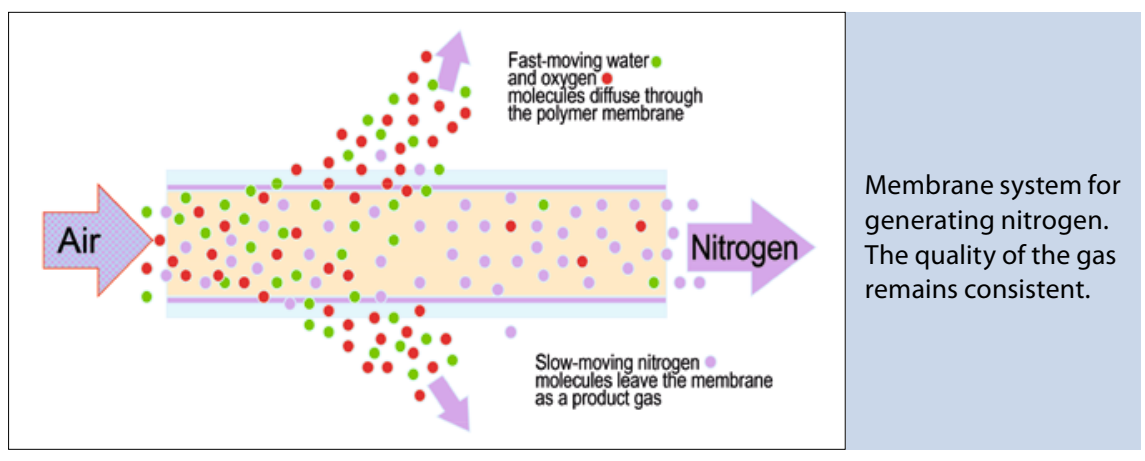

more valuable than material savings. For example, when nitrogen atomisation is used the picture frame effect rarely occurs. This also applies to thick edges. The number of runs is significantly lower and the gloss level of the coating is considerably improved. In addition, it has been possible to reduce the application time and increase the throughput of parts.

Nitrogen (left) versus compressed air (right): The picture on the left shows the first spraying attempt with the atomiser pressure reduced by half and without fine-tuning the application parameters. The coating applied was around $40 \%$ thicker.

The new process also brings benefits with regard to the availability of the paint shop. Productivity can be in-

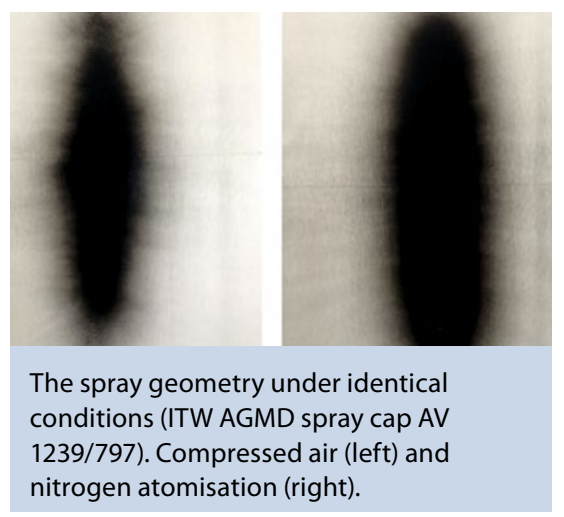

creased as a result of the lower levels of contamination in the booths and the longer service life of the air caps. Another significant advantage of the use of nitrogen in place of compressed air is that the atomiser conditions are always identical.

\section{Successful implementation in practice}

Following on from these findings, the first field tests were carried out in early 2012 with users who require high-quality painted finishes. A number of interesting projects took place which often produced even better results in practice than those achieved during the course of testing.

It repeatedly became clear that, although the reduction in paint consumption is a positive development, the other advantages of the new system were the decisive factor in its introduction. References, in particular from the automotive supply industry, demonstrate the success of the nitrogen application process in volume production. I

\section{Peter Spang}

Oltrogge \& Co KG, Bielefeld, Germany, p.spang@oltrogge.de, www.oltrogge.de 